\title{
Impact behaviour of pressurised pipelines
}

\author{
N. Jones \& R. S. Birch \\ Impact Research Centre, Department of Engineering, \\ University of Liverpool, England, UK
}

\begin{abstract}
Experimental tests are reported on steel pipelines that have been struck by a relatively large rigid wedge-shaped mass travelling up to $10.4 \mathrm{~m} / \mathrm{s}$. A pipeline is supported across a span, is fully clamped at both ends and is struck at the mid-span and at the one quarter span positions. Most of the pipelines are pressurised with a nitrogen gas. The initial impact energy produces large inelastic ductile deformations of the pipeline and, in some cases, failure. The experimental results are compared with previous data obtained on larger diameter pipelines and observations are offered on the accuracy of the geometrically similar scaling of the final deformations. The results are also compared with several empirical equations and comments are made on their accuracy.
\end{abstract}

Keywords: pipelines, impact loading, internal pressure, ductile deformations, failure, empirical equations, geometrically similar scaling.

\section{Introduction}

Pipelines are used throughout industry to convey gases and liquids under high pressures over long distances and between pressure vessels and other industrial plant. These pipelines are often situated in potentially dangerous environments so that safety calculations are required by various bodies to assess the hazards associated with the accidental release of any contents. Impact loads are particularly hazardous and estimates are required for the resistance of a pressurised pipeline to any object that, for example, may be dropped from a crane during maintenance operations, or propelled by the gases after an explosion that causes fragmentation of a pressure vessel. This article focuses on those extreme events when heavy objects travelling at relatively low velocities 
strike a pipeline and cause large inelastic deformations leading to a breach of the integrity for sufficiently severe impact loads.

Some early theoretical studies and experimental results on unpressurised ductile metal pipelines, which are subjected to local static and low-velocity impact loads, are reviewed in References [1-3]. In addition, Brown et al [4] reported on the behaviour of $300 \mathrm{~mm}$ diameter mild steel pipelines impacted with solid missiles having various nose shapes and travelling with speeds up to $14.1 \mathrm{~m} / \mathrm{s}$. Chen and Shen [5] conducted experimental tests on the failure of fully clamped mild steel pipes struck at various locations along the span by wedgeshaped indenters travelling up to $10.69 \mathrm{~m} / \mathrm{s}$. Studies have also been reported on the response of pipelines struck by missiles travelling at impact velocities which are higher than those of interest in this article.

Xiaoqing and Stronge [6], Neilson et al [7] and others explored the influence of internal pressures and various contents on the perforation of pipelines struck by missiles travelling at relatively high velocities. Experimental tests at low velocities up to about $13.6 \mathrm{~m} / \mathrm{s}$ were reported in Reference [8] on pipelines pressurised by a compressible gas (nitrogen). The pipeline specimens were the same as one set of the unpressurised test pipelines in Reference [2]. A wedgeshaped impactor struck normal to the pipeline axis at the mid-span and one quarter span positions and caused permanent inelastic deformations of the pipelines. The integrity of the pipelines was breached underneath the impactor or at a support for sufficiently large impact energies.

The present manuscript extends the study in Reference [8] by reporting some additional experimental results on the low-velocity impact behaviour of pipelines. Comparisons are made with previous experimental data, which leads to some discussion on the accuracy of geometrically similar scaling and recommendations on the choice of empirical equations for design purposes.

\section{Experimental arrangement}

The experimental arrangement used for the current tests is similar to that illustrated in Figure 1 of Reference [8] and is idealised in Figure 1 here. The pipeline has a mean radius $\mathrm{R}=14.5 \mathrm{~mm}$, a thickness $\mathrm{H}=1 \mathrm{~mm}$ and is fully clamped in a fitting which is mounted on the anvil of a drop hammer rig. The free span of all of the pipelines is $2 \mathrm{~L}=300 \mathrm{~mm}$ and they are pressurised through the end clamps with a nitrogen gas. The wedge-shaped missile has a mass $\mathrm{G}$ with an included angle of 15 degrees and has the same shape as that used in Reference [8]. The impact face is $30 \mathrm{~mm}$ wide and $1.5 \mathrm{~mm}$ across with the $30 \mathrm{~mm}$ width orthogonal to the pipeline axis.

\section{Geometrically similar scaling}

The maximum permanent transverse displacement $\left(\mathrm{W}_{\mathrm{f}}\right)$ of a rigid, perfectly plastic pipeline (yield stress $\sigma_{y}$, density $\rho$ ) of length $2 \mathrm{~L}$, mean radius $\mathrm{R}$ and wall thickness $H$, when impacted by a mass $G$ travelling with an initial velocity $V_{o}$, 
can be expressed in terms of four dimensionless $\pi$-terms according to the Buckingham $\pi$-theorem [9]. Taking $\pi_{1}=\mathrm{W}_{\mathrm{f}} / \mathrm{H}$, these four non-unique parameters are $\pi_{2}=\mathrm{GV}_{\mathrm{o}}{ }^{2} \mathrm{~L} / 32 \sigma_{\mathrm{y}} \mathrm{R}^{2} \mathrm{H}^{2}, \pi_{3}=2 \mathrm{R} / \mathrm{H}, \pi_{4}=\mathrm{L} / \mathrm{R}$ and $\pi_{5}=\mathrm{G} / 4 \pi \mathrm{RHL} \rho$. If the material has a uniaxial rupture strain $\varepsilon_{\mathrm{r}}$, and the pipeline has an internal pressure $\mathrm{p}$ and is impacted at a distance $\mathrm{L}_{1}$ from a support, then we have the additional dimensionless terms $\pi_{6}=\mathrm{L}_{1} / \mathrm{L}, \pi_{7}=\varepsilon_{\mathrm{r}}$ and $\pi_{8}=\mathrm{pR} / \sigma_{\mathrm{y}} \mathrm{H}$. The test specimens in Table 1 have $\pi_{3}=29, \pi_{4}=10.34, \pi_{5}=50.1$ and $\pi_{7}=0.075$, while the values for $\pi_{1}, \pi_{2}, \pi_{6}$ and $\pi_{8}$ are given in Table 1 .

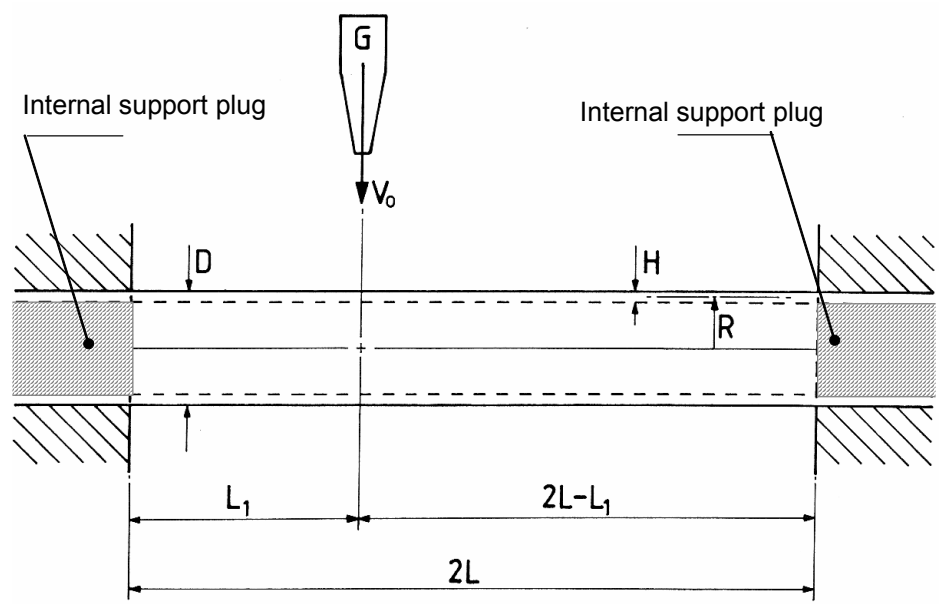

Figure 1: A mass $G$ travelling with an initial velocity $V_{0}$ and striking a pipeline that is fully clamped across a span of $2 \mathrm{~L}$.

\section{Empirical equations}

Several empirical equations have been developed over the years for predicting the final permanent transverse displacements, or damage, of unpressurised pipelines, as noted in References [1,2,8]. The predictions of [10-12] are compared in [3] with the experimental data reported in [3]. Ellinas and Walker [11] with $\mathrm{K}=150$ give good agreement with the experimental data for pipelines with $\pi_{3}=30$ and 40 and struck at the mid-span. Soreide and Amdahl [10] predict good agreement for $\pi_{3}=11$ and 21, while de Oliveira et al [12] predict reasonable agreement with all of the experimental data that was recorded within the range $\pi_{3}$ $=11$ to $\pi_{3}=60$. It was also found that the predictions of Ellinas and Walker [11] overpredicted the value of $\pi_{1}$ for a given value of $\pi_{2}$ for the unpressurised pipelines with $\pi_{3}=35.3$ reported in [8], while their estimates with $\mathrm{K}=300$ gave reasonable agreement with the experimental results. 


\section{Experimental results and discussion}

The experimental results for the dimensionless maximum permanent transverse displacement, $\pi_{1}$, from Table 1 for the unpressurised pipelines, impacted at the mid-span, are plotted in Figure 2 against the dimensionless initial impact energy, $\pi_{2}$, and compared with the corresponding results from [8] having $\mathrm{R}=29.15 \mathrm{~mm}$, $\mathrm{H}=1.7 \mathrm{~mm}$ and $2 \mathrm{~L}=600 \mathrm{~mm}$. The dimensionless term, $\pi_{3}$, is 29 and 34.3 for the experimental results reported in Table 1 and [8], respectively, while $\pi_{4}=10.3$ for both sets of results. The dimensionless mass ratio $\pi_{5}=50.1$ and 14.66 for the two data sets, is much larger than unity, and the difference between the two sets is, therefore, not important from a practical viewpoint since the behaviour of a pipeline is quasi-static for the low impact velocities of interest in the present experimental programme. The variables $\pi_{6}$ and $\pi_{7}$ are identical for both data sets. Also presented in Figure 2 are the unpressurised series E test results with $\pi_{3}=29$ ( $\mathrm{R}=29 \mathrm{~mm}, \mathrm{H}=2 \mathrm{~mm}, 2 \mathrm{~L}=600 \mathrm{~mm}$ ) reported in [2]. It is evident that the three sets of data are not too dissimilar when recognising the differences in the pipeline materials, experimental arrangements and geometrical parameters and when considering the inevitable scatter in tests of this nature.

The specimens in Table 1 are almost one half scale compared to those reported in [8]. It appears that there are no significant departures from the requirements of geometrically similar scaling in terms of the dimensionless variables studied in this manuscript for the low-velocity impact loading of a pipeline, which can be taken as a quasi-static response. Thus, a relationship between $\pi_{1}$ and $\pi_{2}$ should follow the same curve for the two sets of experimental results. It appears from Figure 2 that the results from Table 1 and Reference [8] do lie on a similar curve, but it is unfortunate that the range of $\pi_{2}$ is much higher for the specimens in Table 1 than in [8], so that there is no overlapping between the ranges of the two data sets.

The pressurised pipeline specimens $\left(\pi_{8}>0\right)$ in Table 1 are compared in Figure 3 with those from [8] for impacts at the mid-span $\left(\pi_{6}=1\right)$. The two sets of experimental data encompass a scale range of two and follow roughly the same trend, which is a minimum requirement for geometrically similar scaling. They reveal a tendency for $\pi_{1}$ to depart from linearity with an increase in the dimensionless initial kinetic energy $\pi_{2}$. The critical value of $\pi_{2}$ for the failure of the small-scale pipelines is again more than double that for the larger scale pipelines. It is evident that pipeline failure is sensitive to the magnitude of the internal pressure.

Table 1 contains experimental data for impacts at the one-quarter span location $\left(\pi_{6}=0.5\right)$ on pressurised and unpressurised pipelines. The values of the maximum permanent transverse displacements at the impact point $\left(\pi_{1}\right)$ for ductile behaviour with $\pi_{8}=0$ are somewhat smaller than for the corresponding case in Figure 2 with $\pi_{6}=1$ and $\pi_{8}=0$. It is evident that a catastrophic failure occurs at the clamped support for all of the pressurised test specimens. Moreover, this failure develops for $\pi_{2}<28.0$ when $\pi_{8}=0.26$ and 0.39 , whereas 


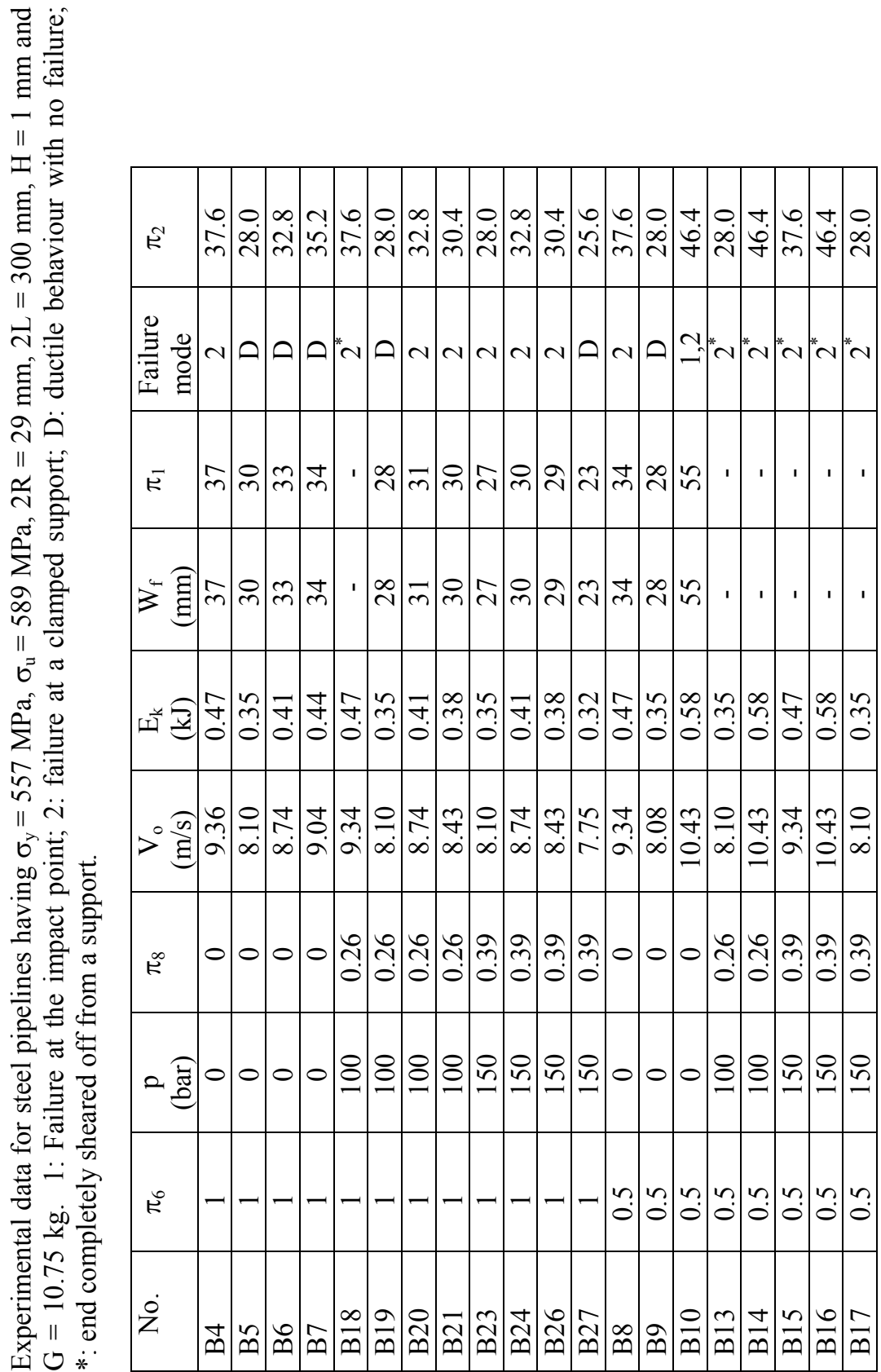

$\frac{\ddot{0}}{\frac{0}{0}}$

WIT Transactions on the Built Environment, Vol 98, (C) 2008 WIT Press www.witpress.com, ISSN 1743-3509 (on-line) 


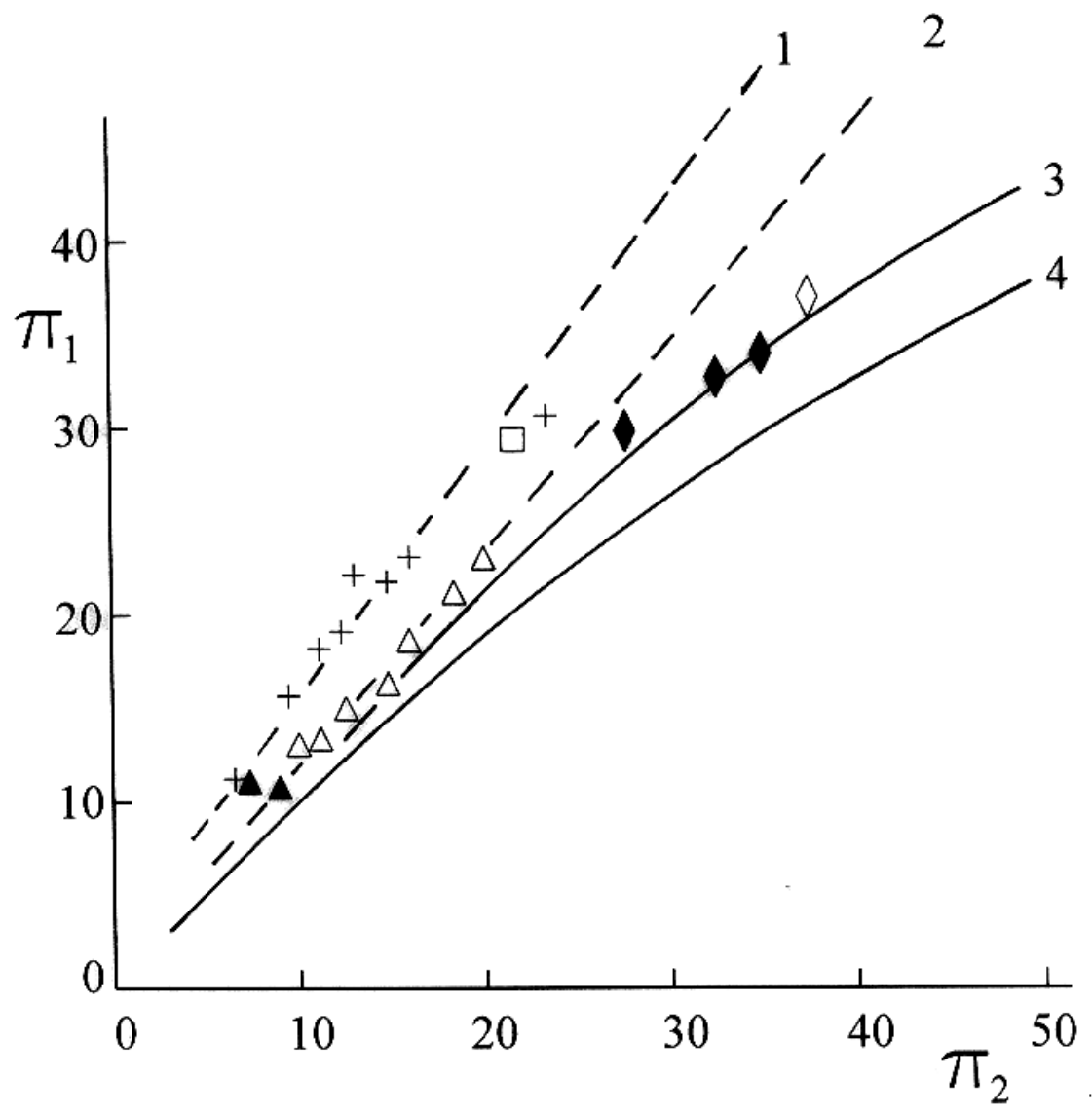

Figure 2: Comparison between experimental results and empirical predictions for the dimensionless maximum permanent transverse displacement, $\mathrm{W}_{\mathrm{f}} / \mathrm{H}$ (i.e., $\left.\pi_{1}\right)$ and the dimensionless initial impact energy $\left(\pi_{2}\right)$ of fully clamped unpressurised $\left(\pi_{8}=0\right)$ steel pipelines struck at the mid-span $\left(\pi_{6}=1\right)$. Experimental results: $\bullet$, large ductile deformations (Table 1); $\diamond$, failure at a clamped support (Table 1); A, large ductile deformations [8]; $\Delta$, local failure at the struck position [8]; $\square$, global failure at a support [8]; +, experimental results [2]. Empirical predictions for the specimens in Table 1:1 and 2, Ellinas and Walker [11] with $\mathrm{K}=150$ and $\mathrm{K}=300$, respectively; 3, de Oliveira et al [12]; 4, Soreide and Amdahl [10].

the response is ductile for this value of $\pi_{2}$ when $\pi_{8}=0$. This behaviour is consistent with the results reported in [8] for pipelines impacted at the one quarter span position. The local and global pipeline failures for impacts at $\pi_{6}=$ 0.5 occur at noticeably smaller values of $\pi_{2}$ than for impacts at the mid-span $\left(\pi_{6}=1\right)$. 


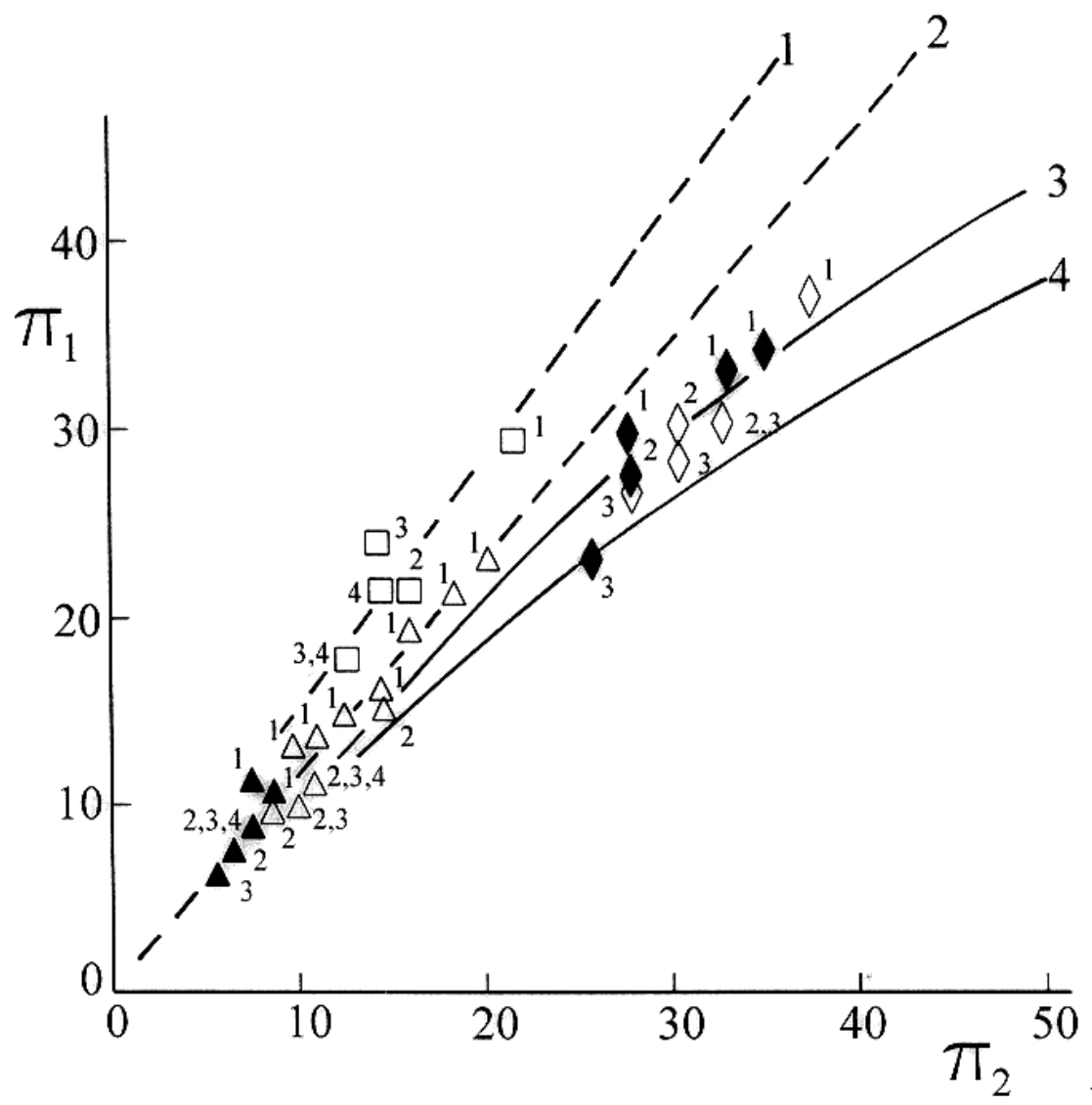

Figure 3: Comparison between experimental results and empirical predictions for the dimensionless maximum permanent transverse displacement, $\mathrm{W}_{\mathrm{f}} / \mathrm{H}$ (i.e., $\left.\pi_{1}\right)$ and the dimensionless initial impact energy $\left(\pi_{2}\right)$ of fully clamped pressurised $\left(\pi_{8} \neq 0\right)$ steel pipelines struck at the mid-span $\left(\pi_{6}=1\right)$. Experimental results: the symbols are defined in Figure 2. The superscripts 1, 2 and 3 refer to $\pi_{8}=0$, 0.26 and 0.39 , respectively, for the specimens in Table 1 . The superscripts $1,2,3$ and 4 refer to $\pi_{8}=0,0.16,0.24$ and 0.32 , respectively, for the specimens [8]. Empirical predictions for $\pi_{8}=$ 0 are defined in Figure 2.

The theoretical predictions of Oliveira et al [12] provides a reasonable estimate and predicts the trend in Figure 2 for the experimental data of Reference [8] and Table 1 having $\pi_{6}=1$ and $\pi_{8}=0$, while Soreide and Amdahl [10] provide a lower bound on the value of $\pi_{1}$ for a given value of $\pi_{2}$. Ellinas and Walker [11] with $K=300$ over-predict $\pi_{1}$ for the results in Table 1 for $\pi_{6}=1$ and $\pi_{8}=0$. This equation does not predict the non-linear trend of the experimental results in the 
$\pi_{1}-\pi_{2}$ space. Nevertheless, it gives good agreement with the results from [8] reported in Figure 2, while Ellinas and Walker [11] with $\mathrm{K}=150$ provides a reasonable estimate of the experimental results from Reference [2]. However, it should be noted that all of the empirical equations in Figure 2 have been calculated for the parameters associated with the pipelines reported in Table 1.

It is evident from Figure 3 that References [10] and [12] again give reasonable predictions for the values of $\pi_{1}$ when $\pi_{8} \neq 0$ and $\pi_{6}=1$. Soreide and Amdahl [10] tend to predict more accurate values at the higher internal gas pressures, while de Oliverira et al [12] give better estimates for the lower internal pressures, so that these two empirical equations tend to bound the experimental results in Figure 3, except, of course, for the global failures. However, neither equation specifically caters for the influence of internal pressure. Ellinas and Walker [11] do not retain the evident non-linearity in Figure 3 so with $\mathrm{K}=300$, and particularly with their recommended value of $K=150$, the predictions, for a given value of $\pi_{2}$, overestimate the value of $\pi_{1}$ for the experimental results in Table 1.

\section{Conclusions}

Large inelastic ductile deformations (damage) of fully clamped steel pipelines are reported for test specimens that are about one-half scale of those reported in [8]. The impact energy causes failure in some cases and releases the internal gas pressure. It transpires that the maximum permanent transverse ductile displacement might satisfy the laws of geometrically similar scaling, but this cannot be shown conclusively because the range of the experimental data does not lie within the range of data in a previous study [8] on larger diameter pipelines. However, a possible contributory factor why the current range of data lies above that in [8] is the observation that the impact energy producing a pipeline failure, which releases the internal gas pressure, does not satisfy the laws of geometrically similar scaling. It is often observed that the dynamic inelastic failure of a full-scale structural member occurs at roughly one half the scaled initial impact energy which would be expected from the scaled results obtained using a one half scale model [9]. The current tests on pipelines confirm this observation.

It is evident from the present results that an increase in the internal gas pressure causes a reduction in the maximum permanent transverse ductile displacement for a given value of the impact energy, as well as causing failure at a smaller impact energy.

The maximum permanent transverse ductile displacement of a pipeline struck at the one quarter span position is smaller than the corresponding value associated with a pipeline struck at the mid-span with the same impact energy. Moreover, failure occurs at smaller impact energies. These observations are consistent with impact studies on beams struck at various locations across the span $[13,14]$.

Several empirical equations, which do not retain internal pressure effects, are compared with the experimental data in Figures 2 and 3. It is revealed that the 
predictions of de Oliveira et al [12] provide a good estimate of the large ductile deformations at the mid-span of both the unpressurised and the pressurised pipelines. Thus, this empirical equation could be used for design purposes, at least on the basis of the range of parameters examined in the current study. The predictions of Soreide and Amdahl [10] provide a lower bound on $\pi_{1}$ for the unpressurised case, but give a good estimate for the higher internal gas pressures. The empirical equation of Ellinas and Walker [11] overpredicts $\pi_{1}$ for the current data in both Figures 2 and 3, though the equations are simple to use and do provide an upper bound for the current results.

\section{Notation}

$\mathrm{p} \quad$ internal pressure

D outside diameter of a pipeline

$E_{k} \quad$ initial kinetic energy of a striker

$\mathrm{G}$ mass of a striker

$\mathrm{H} \quad$ wall thickness of a pipeline

2L span of a fully clamped pipeline

$\mathrm{L}_{1} \quad$ impact location of a striker measured from a clamped support, as shown in Figure 1.

$\mathrm{R}$ mean radius of a pipeline

$\mathrm{V}_{0} \quad$ initial impact velocity of a striker

$\mathrm{W}_{\mathrm{f}} \quad$ maximum permanent transverse displacement of a pipeline

$\varepsilon_{\mathrm{r}} \quad$ engineering rupture strain of a uniaxial tensile test specimen cut from a pipeline.

$\pi_{1}-\pi_{8}$ dimensionless terms defined in the section on geometrically similar scaling

$\rho \quad$ density of the pipeline material

$\sigma_{y}, \sigma_{u}$ uniaxial yield stress and ultimate tensile strength of the pipeline material.

\section{Acknowledgements}

The authors wish to take this opportunity to thank the Impact Research Centre at the University of Liverpool for their cooperation, to Mr. P. McMullon for his assistance with the experimental work and to Mrs. I. M. Arnot for her assistance with the figures.

\section{References}

[1] Reid, S. R., Goudie, K., Denting and bending of tubular beams under local loads. Structural Failure, Ed., T. Wierzbicki and N. Jones, John Wiley, pp 331-364, 1989.

[2] Jones, N., Birch, S. E., Birch, R. S., Zhu, L., Brown, M., An experimental study on the lateral impact of fully clamped mild steel pipes. Proc. I. Mech. E., Vol. 206(E), pp 111-127, 1992. 
[3] Jones, N., Shen, W. Q., A theoretical study of the lateral impact of fully clamped pipelines. Proc. I. Mech. E., Vol. 206(E), pp 129-146, 1992.

[4] Brown, M., Jacobs, M., Mihsein, M., Impact and perforation of mild steel pipes by low velocity missiles. 2nd International Conference on Structures Under Shock and Impact, Ed. P. S. Bulson, Computational Mechanics Publications, Southampton and Boston and Thomas Telford, London, pp 3950, 1992.

[5] Chen, K., Shen, W. Q., Further experimental study on the failure of fully clamped steel pipes. Int. J. of Impact Eng., Vol. 21, No. 3, pp 177-202, 1998.

[6] Xiaoqing, M., Stronge, W. J., Spherical missile impact and perforation of filled steel tubes. Int. J. of Impact Eng., Vol. 3, No. 1, pp 1-16, 1985.

[7] Neilson, A. J., Howe, W. D., Garton, G. P., Impact resistance of mild steel pipes: an experimental study. AEE Winfrith Report R 2125, 1987.

[8] Jones, N., Birch, R. S., Influence of internal pressure on the impact behaviour of steel pipelines. Trans. ASME, Journal of Pressure Vessel Technology, Vol. 118, No. 4, pp 464 471, 1996.

[9] Jones, N., Structural Impact. Cambridge University Press, Cambridge, England, 1989, Paperback edition, 1997.

[10] Soreide, T. H., Amdahl, J., Deformation characteristics of tubular members with reference to impact loads from collision and dropped objects. Norwegian Maritime Research Paper 2, pp 3-12, 1982.

[11] Ellinas, C. P., Walker, A. C., Damage on offshore tubular bracing members. IABSE Colloquium on Ship Collisions with Bridges and Offshore Structures. Copenhagen, pp 253-261, 1983.

[12] de Oliveira, J. G., Wierzbicki, T., Abramowicz, W., Plastic behaviour of tubular members under lateral concentrated loading. Det Norske Veritas Tech. Rep. 82-0708, 1982.

[13] Jones, N., Jones, C., Inelastic failure of fully clamped beams and circular plates under impact loading. Proc. I. Mech. E., Vol. 216(C), pp 133-149, 2002.

[14] Alves, M., Jones, N., Impact failure of beams using damage mechanics: part 2-application. Int. J. of Impact Eng., Vol. 27, No. 8, pp 863-890, 2002. 